\title{
A MONOTONICITY PROPERTY OF RIEMANN'S XI FUNCTION AND A REFORMULATION OF THE RIEMANN HYPOTHESIS
}

\author{
JONATHAN SONDOW ${ }^{1}$ and CRISTIAN DUMITRESCU ${ }^{2}$ \\ ${ }^{1} 209$ West 97th Street, New York, New York 10025, USA \\ Email: jsondow@alumni.princeton.edu \\ ${ }^{2} 119$ Young Street, Kitchener, Ontario, N2H4Z3, Canada \\ Email: cristiand43@gmail.com
}

\begin{abstract}
We prove that Riemann's xi function is strictly increasing (respectively, strictly decreasing) in modulus along every horizontal half-line in any zero-free, open right (respectively, left) half-plane. A corollary is a reformulation of the Riemann Hypothesis.
\end{abstract}

\section{Introduction}

The Riemann zeta function $\zeta(s)$ is defined as the analytic continuation of the Dirichlet series

$$
\zeta(s)=\sum_{n=1}^{\infty} \frac{1}{n^{s}}
$$

which converges if $\mathfrak{R}(s)>1$. The zeta function is holomorphic in the complex plane, except for a simple pole at $s=1$. The real zeros of $\zeta(s)$ are $s=-2,-4,-6, \ldots$ Its nonreal zeros lie in the critical strip $0 \leq \mathfrak{R}(s) \leq 1$. The Riemann Hypothesis asserts that all the nonreal zeros lie on the critical line $\mathfrak{R}(s)=1 / 2$.

Riemann's xi function $\xi(s)$ is defined as the product

$$
\xi(s):=\frac{1}{2} s(s-1) \pi^{-\frac{1}{2} s} \Gamma\left(\frac{1}{2} s\right) \zeta(s)
$$

Mathematics subject classification number: 11M26.

Key words and phrases: critical line, critical strip, functional equation, gamma function, Hadamard product, horizontal half-line, open half-plane; increasing in modulus, monotonicity, nonreal zero, Riemann Hypothesis, Riemann zeta function, xi function. 
where $\Gamma$ denotes the gamma function. The zero of $s-1$ cancels the pole of $\zeta(s)$, and the real zeros of $s \zeta(s)$ are cancelled by the (simple) poles of $\Gamma\left(\frac{1}{2} s\right)$, which never vanishes. Thus, $\xi(s)$ is an entire function whose zeros are the nonreal zeros of $\zeta(s)$ (see [1, p. 80]). The xi function satisfies the remarkable functional equation

$$
\xi(1-s)=\xi(s)
$$

We prove the following monotonicity property of $\xi(s)$. (Throughout this note, increasing and decreasing will mean strictly so, and a half-line will be a half-infinite line not including its endpoint.)

THEOREM 1. The xi function is increasing in modulus along every horizontal half-line lying in any open right half-plane that contains no xi zeros. Similarly, the modulus decreases on each horizontal half-line in any zero-free, open left half-plane.

For example, since $\xi(s) \neq 0$ outside the critical strip, if $t$ is any fixed number, then $|\xi(\sigma+i t)|$ is increasing for $1<\sigma<\infty$ and decreasing for $-\infty<\sigma<0$.

In the next section, as a corollary of Theorem 1, we give a reformulation of the Riemann Hypothesis (a slight improvement of [2, Section 13.2, Exercise 1 (e)]). The proof of Theorem 1 is presented in the final section.

\section{A reformulation of the Riemann Hypothesis}

Here is an easy corollary of Theorem 1.

COROLLARY 1. The following statements are equivalent.

(i). If $t$ is any fixed real number, then $|\xi(\sigma+i t)|$ is increasing for $1 / 2<\sigma<\infty$.

(ii). If $t$ is any fixed real number, then $|\xi(\sigma+i t)|$ is decreasing for $-\infty<\sigma<1 / 2$.

(iii). The Riemann Hypothesis is true.

PROOF. If $|\xi(s)|$ is increasing along a half-line $L$ (or decreasing on $L$ ), then $\xi(s)$ cannot have a zero on $L$. It follows, using the functional equation, that each of the statements (i) and (ii) implies (iii). Conversely, if (iii) holds, then $\xi(s) \neq 0$ on the right and left open half-planes of the critical line, and Theorem 1 implies (i) and (ii).

\section{Proof of Theorem 1}

We prove the first statement. The second then follows, using the functional equation. 
Let $H=H\left(\sigma_{0}\right)=\left\{s: \Re(s)>\sigma_{0}\right\}$ be a zero-free, open right half-plane. Fix a real number $t_{0}$, and denote by $L=L\left(\sigma_{0}, t_{0}\right)$ the horizontal half-line

$$
L=\left\{\sigma+i t_{0}: \sigma>\sigma_{0}\right\} \subset H=\left\{\sigma+i t: \sigma>\sigma_{0}\right\}
$$

In order to prove that $|\xi(s)|$ is increasing along $L$, we employ the Hadamard product representation of the xi function [1, p. 80]:

$$
\xi(s)=\frac{1}{2} e^{B s} \prod_{\rho}\left(1-\frac{s}{\rho}\right) e^{s / \rho}
$$

Here the product is over all nonreal zeta zeros $\rho$, and $B$ is the negative real number

$$
B:=\frac{1}{2} \log 4 \pi-1-\frac{1}{2} C=-0.023095 \ldots,
$$

where $C$ is Euler's constant.

We first prove that $|1-(s / \rho)|$ is increasing on $L$. Since $H=\left\{s: \Re(s)>\sigma_{0}\right\}$ is zero-free and $L \subset H$, we have

$$
\mathfrak{R}(\rho) \leq \sigma_{0}<\mathfrak{R}(s) \quad(s \in L) .
$$

It follows that the distance $|s-\rho|$ and, hence, the modulus $|1-(s / \rho)|=|s-\rho||\rho|^{-1}$ are increasing along $L$.

We next show that $\left|e^{s / \rho}\right|$ is non-decreasing on $L$. (In fact, $\left|e^{s / \rho}\right|$ is increasing on $L$, but we do not need this deeper fact.) Let $\rho=\beta+i \gamma$ denote a nonreal zeta zero. Since $\beta=\Re(\rho) \geq 0$, the modulus

$$
\left|e^{s / \rho}\right|=e^{\Re(s / \rho)}=e^{\left(\beta \sigma+\gamma t_{0}\right) /\left(\beta^{2}+\gamma^{2}\right)}
$$

is non-decreasing along $L$.

It remains to overcome the effect of the Hadamard product factor $e^{B s}$, which, since $B<0$, is decreasing in modulus on $L$. We use the following alternate interpretation of the constant $B$. First, let $\rho_{1}, \rho_{2}, \ldots$ be the zeta zeros with positive imaginary part, and write $\rho_{n}=\beta_{n}+i \gamma_{n}$, for $n \geq 1$. Then $B$ is also given by the formulas [1, p. 82]

$$
B=-\sum_{n=1}^{\infty}\left(\frac{1}{\rho_{n}}+\frac{1}{\bar{\rho}_{n}}\right)=-2 \sum_{n=1}^{\infty} \frac{\beta_{n}}{\beta_{n}^{2}+\gamma_{n}^{2}} .
$$

For $N \geq 1$, denote the $N$ th partial sum of the series for $-B$ by 


$$
S_{N}:=\sum_{n=1}^{N}\left(\frac{1}{\rho_{n}}+\frac{1}{\bar{\rho}_{n}}\right)
$$

Note that $-\left(B+S_{N}\right)$ is positive, and that it approaches zero as $N$ tends to infinity.

Now for $N \geq 2$, let $P_{N}(s)$ be the finite product

$$
P_{N}(s):=\left(1-\frac{s}{\overline{\rho_{1}}}\right) \prod_{n=2}^{N}\left(1-\frac{s}{\rho_{n}}\right)\left(1-\frac{s}{\bar{\rho}_{n}}\right) .
$$

Then by combining exponential factors, we can write the Hadamard product as

$$
\xi(s)=\frac{1}{2} e^{\left(B+S_{N}\right) s}\left(1-\frac{s}{\rho_{1}}\right) P_{N}(s) \prod_{n=N+1}^{\infty}\left(1-\frac{s}{\rho_{n}}\right) e^{s / \rho_{n}}\left(1-\frac{s}{\bar{\rho}_{n}}\right) e^{s / \bar{\rho}_{n}} .
$$

From what we have shown about $|1-(s / \rho)|$ and $\left|e^{s / \rho}\right|$, both $P_{N}(s)$ and the infinite product are increasing in modulus along $L$. To analyze the remaining factors on $L$, set $s=\sigma+i t_{0}$ and define the function

$$
f_{N}(\sigma):=\left|\frac{1}{2} e^{\left(B+S_{N}\right) s}\left(1-\frac{s}{\rho_{1}}\right)\right|^{2}=\frac{1}{4} e^{2\left(B+S_{N}\right) \sigma} \frac{\left(\sigma-\beta_{1}\right)^{2}+\left(t_{0}-\gamma_{1}\right)^{2}}{\beta_{1}^{2}+\gamma_{1}^{2}} .
$$

A calculation shows that the derivative $f_{N}^{\prime}(\sigma)$ is positive if

$$
\frac{\sigma-\beta_{1}}{\left(\sigma-\beta_{1}\right)^{2}+\left(t_{0}-\gamma_{1}\right)^{2}}>-\left(B+S_{N}\right)
$$

Now fix $\sigma_{1}>\sigma_{0}$. Since $\sigma_{1}-\beta_{1} \geq \sigma_{1}-\sigma_{0}>0$, and $-\left(B+S_{N}\right) \rightarrow 0$ as $N \rightarrow \infty$, we can choose $N$ so large that $f_{N}^{\prime}\left(\sigma_{1}\right)>0$. Then $f_{N}^{\prime}$ is also positive on some open interval $I$ containing $\sigma_{1}$. It follows that $f_{N}(\sigma)$ and, therefore, $\left|\xi\left(\sigma+i t_{0}\right)\right|$ are increasing for $\sigma \in I$. Since $\sigma_{1}\left(>\sigma_{0}\right)$ and $t_{0}$ are arbitrary, the theorem is proved.

\section{References}

[1] H. DAvenPort, Multiplicative Number Theory, 2nd ed., revised by H. L. Montgomery, Graduate Texts in Mathematics, 74, Springer-Verlag, New York-Berlin, 1980.

[2] H. L. Montgomery and R. C. Vaughan, Multiplicative Number Theory, I, Classical Theory, Cambridge Studies in Advanced Mathematics, 97, Cambridge University Press, Cambridge, UK, 2007. 